\title{
LPD Based Route Optimization in Nested Mobile Network ${ }^{\star}$
}

\author{
Jungwook Song ${ }^{1}$, Heemin $\mathrm{Kim}^{1}$, Sunyoung Han ${ }^{1, \star \star}$, and Bokgyu Joo ${ }^{2}$ \\ ${ }^{1}$ Department of Computer Science and Engineering, Konkuk University \\ 1 Hwayang, Gwangjin, Seoul 143-701, Korea \\ \{swoogi, procan, syhan\}@konkuk.ac.kr \\ 2 Department of Computer and Information Communications, Hongik University \\ 300 Shinan, Jochiwon, Chungnam 339-701, Korea \\ bkjoo@hongik.ac.kr
}

\begin{abstract}
IETF working groups developed mobile IP protocols to support host mobility, and the NEMO(Network Mobility) Working Group, specifically, developed the NEMO Basic Support Protocol. The NEMO basic solution enables mobile networks to change their point of attachment to the Internet. The protocol, however, leads to suboptimal route, and other problems. The problems become serious when mobile networks are nested. In this paper, we present a route optimization mechanism for nested mobile networks based on 'Limited Prefix Delegation' technique. We present performance evaluation results by simulation to confirm the effectiveness.
\end{abstract}

\section{Introduction}

As the mobile access networks become utility for everyday life, there will be thousands of mobile nodes change their locations simultaneously and, in a city, mobile networks will be common place. IETF standardized NEMO(Network Mobility) Basic Support Protocol(NBSP) 1] to support network mobility by extending MIPv6[2]. With the NBSP, networks as well as hosts can freely change the points of attachment to the Internet, while nodes in the network still preserving their on-going communication sessions to.

The NBSP, however, does not provide optimal routing path of data packets, which results in various problems including packet delay and loss [3], and the problems become serious when mobile networks are nested. The nesting of mobile networks will become common in the future, since lots of networks as well as hosts will change their locations freely. For example, people with personal area network $(\mathrm{PAN})$ ride into a bus with its own mobile network, and buses move into a car-ferry with its own mobile network. Therefore, route optimization of mobile network is major issue to be solved in mobile IP research.

In this paper, we propose a route optimization solution based on 'Limited Prefix Delegation'(LPD) technique. Our solution is an extension of the NBSP

* This research was supported by the 'Seoul R\&D Program'.

${ }^{\star \star}$ Corresponding author. 
by making some modification on mobile router(MR) function. The concept of our solution was presented elsewhere in a short article 4]. In this paper, we complete the concept by performing a comprehensive analysis of the mechanism and presenting simulation results. By simulation we showed the effectiveness of our solution compared to NBSP. We also analyze our mechanism for real situations that may arise during the deployment stage.

\section{LPD-Based Route Optimization}

The problem of NBSP become serious if mobile networks are nested. As the nesting level increases, so is the number of bi-directional tunnels between MRs and their HAs. The route of data packets become longer and complicated(called 'pinball routing problem'). Moreover, the needs for multiple encapsulation(deep tunnels) increases the processing overhead at HA and MR, as well as the header size. The effects of sub-optimal route of the NBSP are fully described in [3].

\subsection{Limited Prefix Delegation Technique}

Our LPD mechanism added and modified some features to the NBSP as follows:

1. Add a new RA(Router Advertisement) option

2. Extend the binding update procedure at the mobile router

3. Modify tunneling process at the mobile router

In our mechanism, key concept is the delegation of the access router's prefix to the MRs underneath. The effect is that only one MR in the nested mobile network opens direct tunnel to the $\mathrm{CN}$, and route optimization is achieved.

For the MR that is attached to the access router directly, the delegation is simple. To achieve this, we add new RA option called 'delegated prefix option' to RA message. MNNs other than MR silently ignore this option. This way, all MRs in the nested mobile network will have care-of addresses that is the subnet address of the access router of visited network. More detail mechanism is described in 4 .

\subsection{Analysis of Routes Taken by LPD Mechanism}

Please refer to the Appendix B of [3] in parallel with this section. Then, we consider special situation during deployment stage of this mechanism and show that ours still achieves optimal route practically possible. Analysis of all cases(case A to L) are summarized in Table 1.

During deployment stage of LPD mechanism, however, we could not expect that all MRs in nested mobile networks are LPD-extended, i.e., some MRs are just 'plain MR' supporting only NBSP and some are 'LPD-extended MR'. Our solution still achieves near-optimal route even in that circumstance.

In the Case B of [3, if all MRs are 'plain MR' and CN is Mobile IPv6-enabled, the routing path of packets between VMN and CN by NBSP would be taken 
Table 1. Routing Path of Each Case with LPD. For example, the Case A - both MNN and $\mathrm{CN}$ are fixed nodes with no mobility functions. In our solution, MR3 opens a tunnel to CN directly, so that packets from the LFN to CN takes an optimized route. Since CN has no mobility function, it sends packets to MR3_HA, then MR3_HA opens tunnel to MR3 directly.

\begin{tabular}{cl}
\hline CASE & Routing Path of LPD Mechanism \\
\hline $\mathrm{A} \quad$ & $\begin{array}{l}\text { (to } C N) \mathrm{LFN} \rightarrow \mathrm{MR} 3 \rightarrow \mathrm{MR} 2 \rightarrow \mathrm{MR} 1 \rightarrow \mathrm{CN} \\
\text { (from } C N) \mathrm{LFN} \leftarrow \mathrm{MR} 3 \leftarrow \mathrm{MR} 2 \leftarrow \mathrm{MR} 1 \leftarrow \mathrm{MR} 3 \_\mathrm{HA} \leftarrow \mathrm{CN}\end{array}$ \\
\hline $\mathrm{B} \quad \mathrm{VMN} \leftrightarrow \mathrm{MR} 3 \leftrightarrow \mathrm{MR} 2 \leftrightarrow \mathrm{MR} 1 \leftrightarrow \mathrm{CN}$ \\
\hline $\mathrm{C} \quad($ to $C N) \mathrm{VMN} \rightarrow \mathrm{MR} 3 \rightarrow \mathrm{MR} 2 \rightarrow \mathrm{MR} 1 \rightarrow \mathrm{CN}$ \\
$\quad($ from $C N) \mathrm{VMN} \leftarrow \mathrm{MR} 3 \leftarrow \mathrm{MR} 2 \leftarrow \mathrm{MR} 1 \leftarrow \mathrm{VMN} \_\mathrm{HA} \leftarrow \mathrm{CN}$ \\
\hline $\mathrm{D}, \mathrm{E} \quad \mathrm{LFN}(\mathrm{VMN}) \leftrightarrow \mathrm{MR} 3 \leftrightarrow \mathrm{MR} 2 \leftrightarrow \mathrm{MR} 1 \leftrightarrow \mathrm{MR} 4 \leftrightarrow \mathrm{MR} 5 \leftrightarrow \mathrm{CN}$ \\
\hline $\mathrm{F} \quad \mathrm{VMN} \leftrightarrow \mathrm{MR} 3 \leftrightarrow \mathrm{MR} 2 \leftrightarrow \mathrm{MR} 1 \leftrightarrow \mathrm{MR} 4 \leftrightarrow \mathrm{MR} 5 \leftrightarrow \mathrm{CN}$ \\
\hline $\mathrm{G}, \mathrm{H} \quad \mathrm{LFN}(\mathrm{VMN}) \leftrightarrow \mathrm{MR} 3 \leftrightarrow \mathrm{MR} 2 \leftrightarrow \mathrm{MR} 1 \leftrightarrow \mathrm{MR} 4 \leftrightarrow \mathrm{MR} 5 \leftrightarrow \mathrm{CN}$ \\
\hline $\mathrm{I}$ & $\mathrm{VMN} \leftrightarrow \mathrm{MR} 3 \leftrightarrow \mathrm{MR} 2 \leftrightarrow \mathrm{MR} 1 \leftrightarrow \mathrm{MR} 4 \leftrightarrow \mathrm{MR} 5 \leftrightarrow \mathrm{CN}$ \\
\hline $\mathrm{J}, \mathrm{K}$ & $\mathrm{LFN}(\mathrm{VMN}) \leftrightarrow \mathrm{CN}$ \\
\hline $\mathrm{L}$ & $\mathrm{VMN} \leftrightarrow \mathrm{MR} 3 \leftrightarrow \mathrm{CN}$ \\
\hline
\end{tabular}

pinball routing(see [3], the Case B of the Appendix B). In the situation when both types of MRs co-exist in this case, there are two cases: two of three MRs are LPD-extended(Case I) and only one of three MRs is LPD-extended(Case II). The routing path of data packets summarized in Table 2.

Table 2. Routing Path of Special Cases. These results show that our solution still achieves near-optimal route possible in any situation.

\begin{tabular}{ll}
\hline CASE & Routing Path of LPD Mechanism \\
\hline Special Case I & $\mathrm{VMN} \leftrightarrow \mathrm{MR} 3 \leftrightarrow \mathrm{MR} 2 \leftrightarrow \mathrm{MR} 1 \leftrightarrow \mathrm{MR} 1 \_\mathrm{HA} \leftrightarrow \mathrm{CN}$ \\
Special Case II & $\mathrm{VMN} \leftrightarrow \mathrm{MR} 3 \leftrightarrow \mathrm{MR} 2 \leftrightarrow \mathrm{MR} 1 \leftrightarrow \mathrm{MR} 1 \_\mathrm{HA} \leftrightarrow \mathrm{MR} 3 \_\mathrm{HA} \leftrightarrow \mathrm{CN}$ \\
\hline
\end{tabular}

\section{Performance Evaluation}

To evaluate the performance of our solution, we performed simulation with OM$\mathrm{NeT}++$, which is open architecture simulation environment for communication network [5]. We considered all twelve cases(A to L) of network configurations specified in the Appendix B of [3. We also performed simulation on two special cases described above. The results of simulations are summarized in Table 3 and Table 4. 
Table 3. Simulation Results(Average RTT). This table shows the statistical results of RTT values for ten configuration model cases. The average RTT values of the LPD mechanism significantly smaller than those of the NBSP for all cases.

\begin{tabular}{ccc||ccc}
\hline Case & NBSP & LPD & Case & NBSP & LPD \\
\hline A & $429 m s$ & $218 m s$ & F & $750 m s$ & $120 m s$ \\
B & $429 m s$ & $114 m s$ & G, H & $753 m s$ & $18 m s$ \\
C & $533 m s$ & $114 m s$ & I & $858 m s$ & $18 m s$ \\
D, E & $645 m s$ & $120 m s$ & L & $858 m s$ & $6 m s$ \\
\hline
\end{tabular}

Table 4. Simulation Results of Special Cases(Average RTT). We also did simulation for the special cases that may arise during deployment. This result shows that even one LPD-enabled MR can decrease network cost.

\begin{tabular}{cc}
\hline Case & Mean of RTTs \\
\hline NBSP A & $429 m s$ \\
Special Case I & $219 m s$ \\
Special Case II & $324 m s$ \\
\hline
\end{tabular}

\section{Concluding Remarks}

In this paper, we proposed the LPD mechanism to solve the route optimization problem of mobile networks that delegates the network prefix of access router to all MRs attached behind. To achieve route optimization, we made some extension to the MR only. In our solution, no change is necessary to other network elements, like MNN, CN, and HA. We need more investigation on security issues to complete the protocol. We are planning to implement our solution on NEPL [6].

\section{References}

1. V. Devarapalli, R. Wakikawa, A. Retrescu, P. Thubert: "Network Mobility (NEMO) Basic Support Prototol", RFC3963, IETF, 2005

2. D. Johnson, C. Perkins, J. Arkko: "Mobility Support in IPv6", RFC3775, 2004

3. C. Ng, P. Thubert, M. Watari, F. Zhao: "Network Mobility Route Optimization Problem Statement", IETF nemo WG Draft, "draft-ietf-nemo-ro-problemstatement-03", 2006

4. Jungwook Song, Sunyoung Han, Kiyong Park: "Route Optimization in NEMO Environment with Limited Prefix Delegation Mechanism", ICCS2006, Part I, LNCS 3991, pp. 936-939, 2006

5. András Varga: OMNeT++: http://www.omnetpp.org

6. Mobile IPv6 for Linux(MIPL): http://www.mobile-ipv6.org/ 